\title{
Investigation of Novel Noncontacting Measurement Method by the Design of Loop-Type Probe and Reconstruction of Radiation Modeling
}

\author{
Sung-Mao Wu, ${ }^{1}$ Cheng-Fu Yang, ${ }^{2}$ Yin-Hsiu Yeh, ${ }^{1}$ Hsin-Fang Li, ${ }^{1}$ and Ren-Fang Hsu ${ }^{1}$ \\ ${ }^{1}$ Micro Electrical Packaging Laboratory, Department of Electrical Engineering, National University of Kaohsiung, \\ Kaohsiung 811, Taiwan \\ ${ }^{2}$ Department of Chemical and Materials Engineering, National University of Kaohsiung, Kaohsiung 811, Taiwan \\ Correspondence should be addressed to Cheng-Fu Yang; cfyang@nuk.edu.tw
}

Received 22 August 2014; Accepted 12 October 2014

Academic Editor: Stephen D. Prior

Copyright @ 2015 Sung-Mao Wu et al. This is an open access article distributed under the Creative Commons Attribution License, which permits unrestricted use, distribution, and reproduction in any medium, provided the original work is properly cited.

\begin{abstract}
Because the ICs' application frequency and speed become higher and trends of system packaging and device under test request higher reliability, a novel technology combining noncontacting measurement method and reconstructing radiation model was proposed to solve signal deliveries in system packages or PCBs. In this study, a novel noncontacting method for the ICs' measurements was investigated by the design of loop-type near-field probe and reconstructed the radiation model to substitute the traditional measurement methods, such as using probes and SMA connectors. A near-field probe was used to receive the coupling signal. The assessing circuit modeling could be completed by some synthesized theorems. According to the study's results, frequency responses of reconstruction model developed by theorems, radiation measurements, and simulated by EM methods were highly curve fitting.
\end{abstract}

\section{Introduction}

Recently, the markets and applications for advanced semiconductor devices' packaging and measurement technologies increase expeditiously. The traditional contacting probes have numerous disadvantages, including low mobility, high cost, and high damaged rate. Table 1 displays several types of probes and probe pins in different ways, which show the specific advantages that they have [1-9]. For example, a device under test (DUT) is a device that is tested to determine the performance and proficiency and is used to check the defects for making sure that the devices can work. The spring contact probes can reduce the pressure to avoid damaging a DUT when the probes touch. Today, measuring the high-density interconnection signals in ICs using large amount contacting probes is a significant issue needed being improved. The most widespread devices for contacting measurements are the vector network analyzer (VNA), automatic test equipment (ATE), and probe station.
Even these contacting probes are usually used for ICs' measurement; they have many unavoidable disadvantages. First, as mentioned above, the ICs become smaller; the contacting probes or SMA connectors are not easily used for measurements, because the reduction in sizes of the probes and SMA connectors is very difficult. The second issue is that, as the measurements are done without sufficient carefulness, contacting processes will usually damage the expensive probes during DUT's process, leading to significant expense at all time. Third, because the contacting probes have low mobility and uniform calibration, the measurement will take a long time. Every time the contacting probes are moved to a new measurement position, they will leave the damage or defects after a DUT on the surface of the measured devices. That will increase the ratio of devices' damage, which will increase the measurements' cost. For those reasons, many researches have focused on the noncontacting measurement methods to find a low-cost and easily fabricated measurement process. 
TABLE 1: Six package designs for investigating electrical effects.

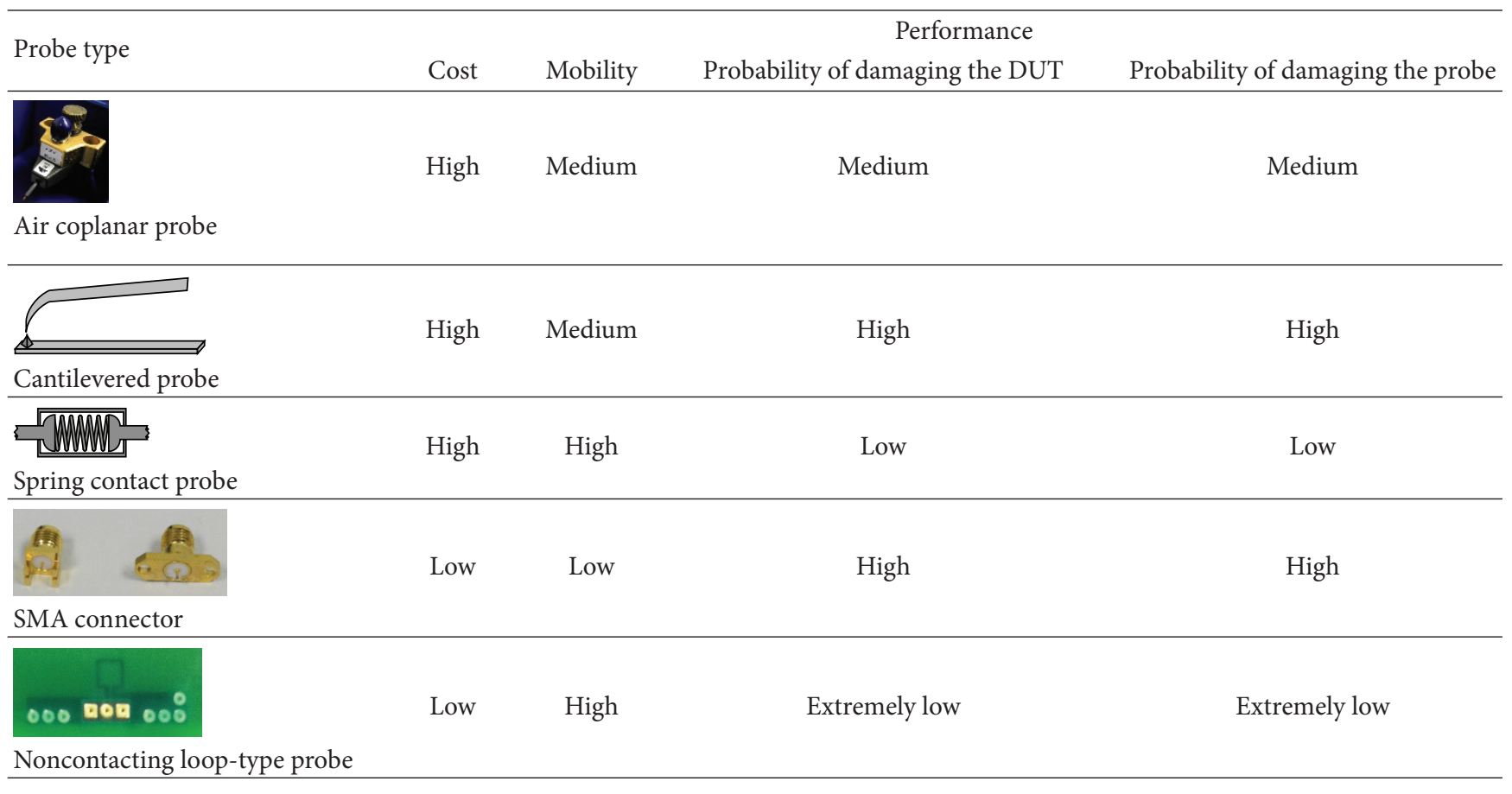

For that, the non-contacting-probe technologies are designed efficiently with broadband, well reproducibility, well directivity, and low loss coupling, respectively. Thus, the noncontacting-probe technologies can transmit or receive signals between different chips or integrated circuits (ICs) in 3D ICs' applications, and it is established physical model up to $10 \mathrm{GHz}$ with coupling effect and loop effect to restore the signal radiation. Because the high frequency of $10 \mathrm{GHz}$ is required, the devices' measurements need to be narrower to keep the measurement data remaining precisely. The area of the noncontacting loop is only about $1.8 \mathrm{~mm} \times 1.8 \mathrm{~mm}$ and the size can be reduced by a precise process, making the loop small enough to measure tiny DUTs. When a DUT or the measurement position is changed, the noncontacting probes can be horizontally moved in rapid speed. Because E-field probe is suitably used in far-field's measurements and $\mathrm{H}$ probe is suitably used in near-field's measurement, the $\mathrm{H}$ probe is used in this study to investigate the noncontacting measurement technique. Because of having high mobility, the noncontacting probes can measure ICs efficiently, and then the needed cost can be reduced. In this study, the near-field probes were used to receive the coupling signal and the circuit modeling could be completely assessed by some synthesized theorems. The differences compared from the measurement results and numerical analysis could be used to validate the feasibility of signal reconstruction.

\section{Design and Structure of Noncontacting Probe}

2.1. Design the Loop-Type Probe. Recently, many kinds of non-contacting-probe measurement technologies had been investigated [3-9], such as coplanar waveguide (CPW) looptype [10], double loop-type [11], and symmetrical square loop-type [12], respectively. For the noncontacting measurements, the most important thing is to design the loop, which has broadband impedance matching and high resolution. Those characteristics can make the loop receive the coupling signals efficiently. Because of the requirement of high reliability, the design structure of signal coupler with the characteristics of broadband, well directivity, and low loss is needed. As indicated above, CPW structure has the features of impedance matching and high bandwidth, and it can enhance the efficiency of the probes. For that, the CPW loop-probe circuits were used to investigate the novel noncontacting structure in this study.

When the noncontacting probes are above DUT equipment, as Figure 1 shows, its input impedance can be calculated by the transmission line method. First, the return loss $\left(S_{11}\right)$ is obtained by the actual measurement data. If we assume $S_{11}=a+j b$, where $a$ and $b$ are the real and imaginary parts of $S_{11}$ value, respectively [13]. Consider

$$
S_{11}=\frac{Z_{\text {in } 1}-Z_{0}}{Z_{\text {in } 1}+Z_{0}}=a+j b,
$$

where $Z_{\text {in } 1}, Z_{\text {in2 }}$, and $Z_{\text {in3 }}$ have their own real and imaginary parts, and $Z_{\mathrm{in} 1}=R_{\mathrm{in} 1}+j X_{\mathrm{in} 1}, Z_{\mathrm{in} 2}=R_{\mathrm{in} 2}+j X_{\mathrm{in} 2}$, and $Z_{\text {in3 }}=R_{\text {in } 3}+j X_{\text {in } 3}$, respectively. After calculation, the probes' impedance can easily be obtained from formula (2). $A$ in 


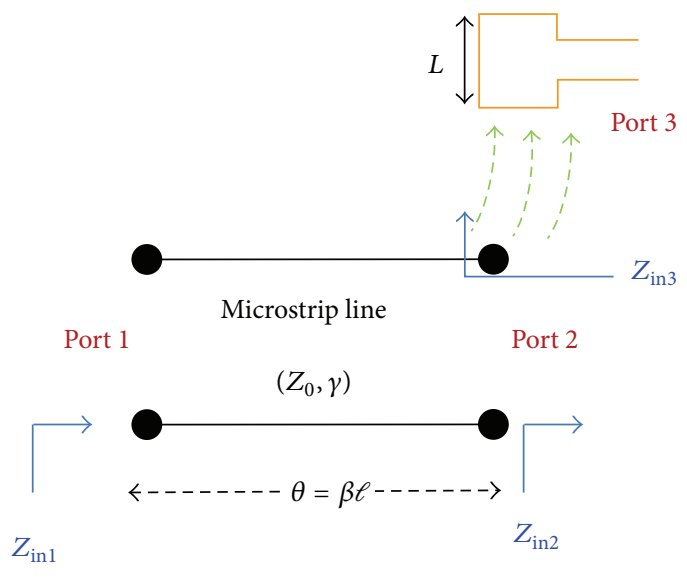

FIGURE 1: Schematic diagram of calculated impedance formula [3].

formulas (3) and (4) is $\tan \beta l$ and $B$ is $Z_{0} R_{\mathrm{in} 2}$, respectively. One has

$$
\begin{aligned}
& Z_{\mathrm{in} 3}=\frac{-Z_{0} Z_{\mathrm{in} 1} Z_{\mathrm{in} 2}+j Z_{\mathrm{in} 2} Z_{0}^{2} A}{Z_{0} Z_{\mathrm{in} 1}-Z_{0} Z_{\mathrm{in} 2}+j A\left(Z_{\mathrm{in} 1} Z_{\mathrm{in} 2}-Z_{0}^{2}\right)} \\
& R_{\mathrm{in} 3}=B\left(\left(-Z_{0}\left|Z_{\mathrm{in} 1}\right|^{2}+Z_{0} R_{\mathrm{in} 1} R_{\mathrm{in} 2}\left(1+A^{2} Z_{0}\right)\right.\right. \\
& \left.-A Z_{0}^{2}\left(2 X_{\mathrm{in} 1}-A Z_{0}\right)\right) \\
& \times\left(\left(Z_{0} R_{\mathrm{in} 1}-Z_{0} R_{\mathrm{in} 2}-A X_{\mathrm{in} 1} R_{\mathrm{in} 2}\right)^{2}\right. \\
& \left.\left.+\left(Z_{0} X_{\mathrm{in} 1}+A R_{\mathrm{in} 1} R_{\mathrm{in} 2}-A Z_{0}^{2}\right)^{2}\right)^{-1}\right) \\
& X_{\mathrm{in} 3}=B\left(\left(A R_{\mathrm{in} 2}\left|Z_{\mathrm{in} 1}\right|^{2}+Z_{0} R_{\mathrm{in} 2}\left[X_{\mathrm{in} 1}\left(1-A^{2}\right)-A Z_{0}\right]\right)\right. \\
& \times\left(\left(Z_{0} R_{\mathrm{in} 1}-Z_{0} R_{\mathrm{in} 2}-A X_{\mathrm{in} 1} R_{\mathrm{in} 2}\right)^{2}\right. \\
& \left.\left.+\left(Z_{0} X_{\mathrm{in} 1}+A R_{\mathrm{in} 1} R_{\mathrm{in} 2}-A Z_{0}^{2}\right)^{2}\right)^{-1}\right) .
\end{aligned}
$$

The design of noncontacting measurement probes with this impedance in formulas (2)-(4) was made of CPW structure, which consisted of feed-line and square loop. There are some advantages for us using CPW structure to design the noncontacting circuits. First, as in Figure 2, because the loop $(S)$ and the reference ground $(G)$ are on the same plane, the probe can reduce the size of the contact area. Second, although the medium between the feed-line and the ground is changed, the characteristic impedance has no apparent variation. Third, the feed-line and the reference ground are close to each other; the radiation loss can be easily decreased. As the specific structure of noncontacting probe is shown in Figure 3, the transmission line of square loop was $1.8 \mathrm{~mm}$ in length and $0.2 \mathrm{~mm}$ in width; the feed-line was $1 \mathrm{~mm}$ in length and $0.3 \mathrm{~mm}$ in width. The detailed data are shown in Table 2.
TABLE 2: Information of the noncontacting loop-type probe.

\begin{tabular}{lccc}
\hline Object & Length & Width & Spacing \\
\hline Feed-line & $1 \mathrm{~mm}$ & $0.3 \mathrm{~mm}$ & $0.15 \mathrm{~mm}$ \\
Loop & $1.8 \mathrm{~mm}$ & $0.2 \mathrm{~mm}$ & \\
\hline
\end{tabular}

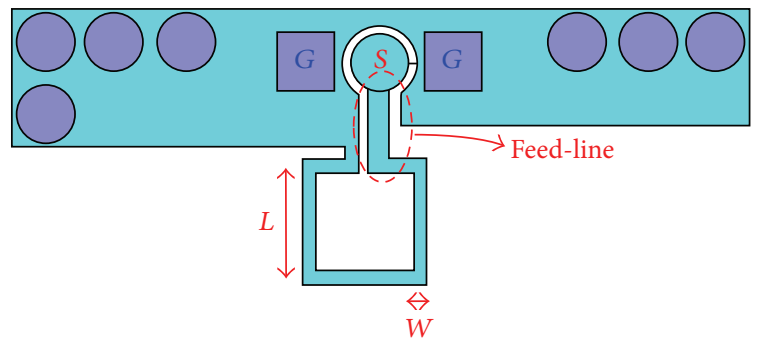

FIGURE 2: Structure of the CPW loop-type probe.

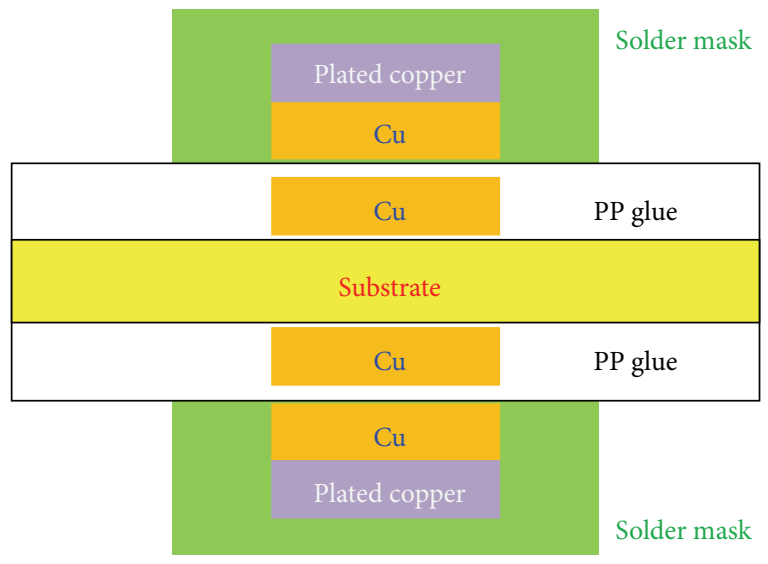

FIGURE 3: Structure of the four-layer stacked substrate in this paper.

2.2. The Process Specification of Loop-Type Probe. The measurements for the board samples were formed by stacking the different materials in a four-layer structure. As Figure 3 shows, the materials for four layers were copper, solder mask, PP glue, and plated copper, respectively. The information of the four-layer materials is shown in Table 3. As from top layer to lowest one, the first layer was a pad with thickness of $0.043 \mathrm{~mm}$; the second and third layers were the looptype probe and the DUT, and both of their thicknesses were $0.035 \mathrm{~mm}$; the fourth layer was GND with thickness of $0.043 \mathrm{~mm}$; thicknesses of the copper plated on layer 1 and layer 4 were $0.025 \mathrm{~mm}$; the thickness of solder mask was $0.053 \mathrm{~mm}$, that of PP glue was $0.15 \mathrm{~mm}$, and that of FR4 was $0.7 \mathrm{~mm}$, respectively.

\section{Reconstruction Theory}

3.1. Derivation. As the transmission line is revealed in Figure 4 and $5.3 \mathrm{GHz}$ band pass filter is revealed in Figure 5, a radiation model can be obtained from two DUTs; one is transmission line and the other is $5.3 \mathrm{GHz}$ filter. The loop is measured and extracted by equivalent lump elements, such as resistance, capacitance, and inductance, respectively. 
TABLE 3: Material thickness information.

\begin{tabular}{lcccc}
\hline \multicolumn{3}{c}{ Copper } & \multicolumn{2}{c}{ Material } \\
\hline Layer 1 & Pad & $0.043 \mathrm{~mm}$ & Solder mask & $0.053 \mathrm{~mm}$ \\
Layer 2 & Loop & $0.035 \mathrm{~mm}$ & PP glue & $0.15 \mathrm{~mm}$ \\
Layer 3 & DUT & $0.035 \mathrm{~mm}$ & Substrate (FR4) & $0.7 \mathrm{~mm}$ \\
Layer 4 & GND & $0.043 \mathrm{~mm}$ & Plated copper & $0.025 \mathrm{~mm}$ \\
\hline
\end{tabular}

TABLE 4: Parameters of the noncontacting measurement model (DUT: transmission line).

\begin{tabular}{lcc}
\hline \multirow{2}{*}{ Resistance } & $\mathbf{R}_{\mathbf{a}}$ & $18 \mathrm{~m} \Omega$ \\
& $\mathbf{R}_{\mathbf{m}}$ & $6.8 \mathrm{k} \Omega$ \\
\hline \multirow{2}{*}{ Capacitance } & $\mathbf{C}_{\mathbf{a}}$ & $0.42 \mathrm{pF}$ \\
& $\mathbf{C}_{\mathbf{m}}$ & $0.07 \mathrm{pF}$ \\
\hline \multirow{2}{*}{ Inductance } & $\mathbf{L}_{\mathbf{a}}$ & $5.8137 \mathrm{nH}$ \\
& $\mathbf{L}_{\mathbf{m}}$ & $2.5 \mathrm{nH}$ \\
\hline
\end{tabular}

TABLE 5: Parameters of the noncontacting measurement model (DUT: three-dimensional stacked for $5.3 \mathrm{G}$ filter).

\begin{tabular}{lcc}
\hline \multirow{2}{*}{ Resistance } & $\mathbf{R}_{\mathbf{a}}$ & $18 \mathrm{~m} \Omega$ \\
& $\mathbf{R}_{\mathbf{m}}$ & $4.9 \mathrm{k} \Omega$ \\
\hline \multirow{2}{*}{ Capacitance } & $\mathbf{C}_{\mathbf{a}}$ & $0.42 \mathrm{pF}$ \\
& $\mathbf{C}_{\mathbf{m}}$ & $0.06094 \mathrm{pF}$ \\
\hline \multirow{2}{*}{ Inductance } & $\mathbf{L}_{\mathbf{a}}$ & $5.8137 \mathrm{nH}$ \\
& $\mathbf{L}_{\mathbf{m}}$ & $2.5 \mathrm{nH}$ \\
\hline
\end{tabular}

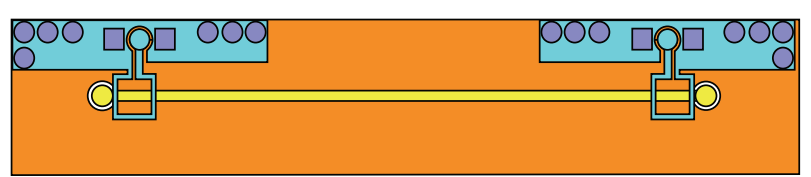

Figure 4: Transmission line measurement.

From the measured $S$-parameters, we will show that the simulation, measurement, and modeling results are matched. Then, the coupling characteristics between the loop-type probe and DUT can be found [14]. The equivalent model and the parameters of the model in different DUTs are shown in Figure 6. The test fixture's effect will exist in the DUTs. However, we have measured and eliminated the test fixture's by calibration with short, open, load, through (SOLT) method.

In order to obtain the parameters of the modeling structure, formulas (5)-(10) are used to calculate the relation from DUT to loop and the obtained results are shown in Tables 4 and $5[15,16]$. Formula (5) is used to calculate the resistance $R$ and Figure 7(a) shows the structure for calculation. The resistance can be obtained by resistivity of using material to multiply the total length $L$ of the probe and to divide by the cross-section area $A_{1}$. Formulas (6) and (7) represent the capacitance formed between the ground- $(G-)$ signal $(S)$ gap of the CPW loop and Figure 7(b) shows the structure of capacitance. A capacitance can be obtained by dielectric constant of using material to multiply the parallel area $A_{2}$ of

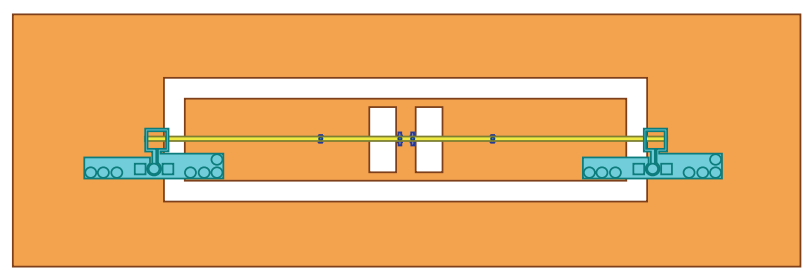

FIGURE 5: Three-dimensional stacked structure for $5.3 \mathrm{G}$ filter's measurement.

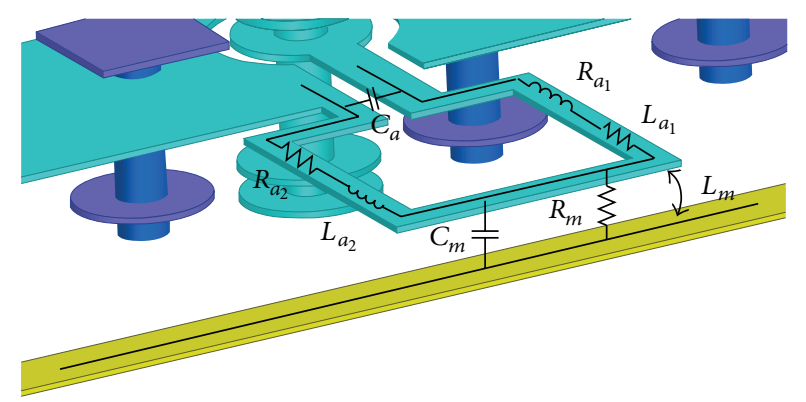

FIGURE 6: Noncontacting measurement model.

used electrode area and to divide by the medium's thickness d. Consider

$$
\begin{aligned}
R & =\rho \frac{L}{A_{1}}(\Omega) \\
C_{a} & =\varepsilon_{r} \varepsilon_{0} \frac{A_{2}}{d}(\text { Farad }) \\
C_{m} & =\frac{A \varepsilon}{d}+\int_{x 1}^{x 2} \frac{W \varepsilon}{\sqrt{d^{2}+x^{2}}} d x .
\end{aligned}
$$

However, the self-inductance $L_{a}$ and mutual inductance $L_{m}$ of the CPW loop can be calculated from formulas (8)(10). The constant $N$ and $I$ are the loop number of coil and the current on the path of coil. From formula (10) and the structure in Figure 8(b), the coupling area $A$ is obtained from the parallel edge areas, and the distance $d$ is the distance between loop and DUT. Consider the following:

$$
\begin{gathered}
L_{a}=2 \ell\left(\ln \frac{2 \ell}{w+t}+0.50049+\frac{w+t}{3 \ell}\right) \\
B=\frac{2 \sqrt{2} \mu_{0} I}{\pi \ell} \\
L_{m}=\frac{N B S}{I} \text { (Henry). }
\end{gathered}
$$

Therefore, the signals in noncontacting probe are obtained by the coupling method, which is different from traditional measurement method by using probes' contacting process. For that, a complete reduction theory to compensate the being coupled signals measured by the noncontacting probes is needed. The reduction theory for the noncontact probe not only restores the characteristics of the DUT but also creates the similar data with those of the traditional contacting probes. As shown in Figure 9, each alphabet represents a part of the physical model, while each number represents that the position it is in of the circuit. 


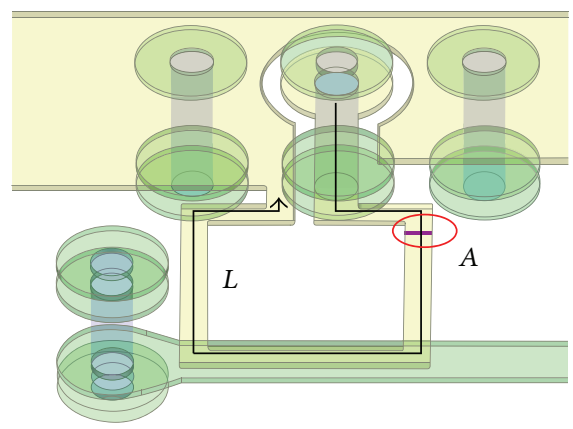

(a)

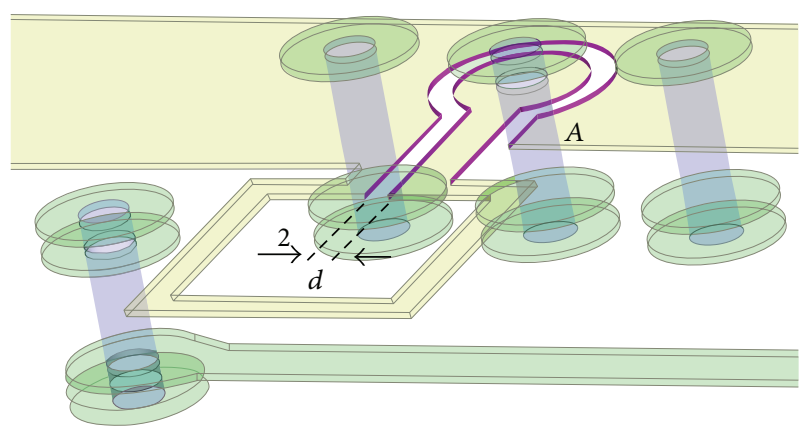

(b)

FIgURE 7: Probe parameters calculation. (a) Resistance and (b) capacitance.

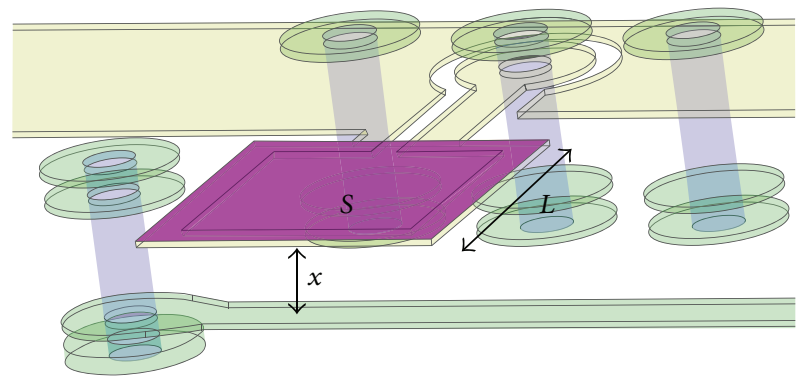

(a)

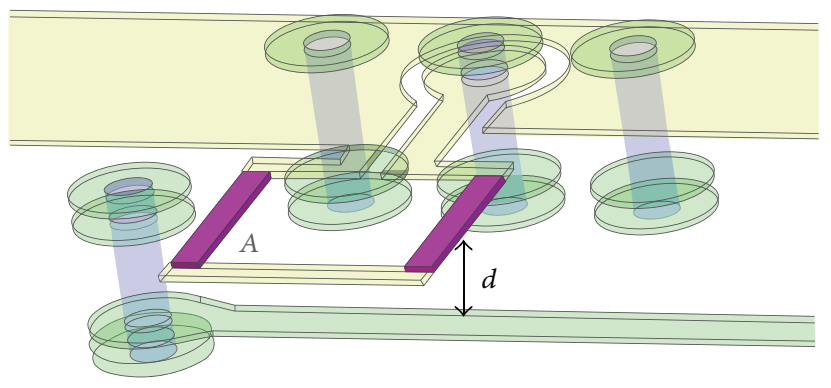

(b)

FIGURE 8: Parameters between loop and DUT calculation. (a) Mutual inductance and (b) dielectric capacitance.

If we set point 2 in Figure 9 as the reference point, then we can calculate the input impedance $\left(Z_{\text {in }}\right)$ from point $A$ to point 2 shown in Figure 9. When point $a$ is connected to point $b$ in shunt, then formula (12) is obtained. Under the same process, point $c$ and point $d$ are connected in shunt, and they can connect in series with $h$ to obtain formula (13). As the same theorem is used, as point $e$ and point $f$ are connected in shunt, they can connect with $i$ to obtain formula (14). Since the model shown in Figure 9 is symmetrical, $Z_{\text {in }}$ can be calculated by multiplying $j$ by twice, minus $a$ by once, and then being added to $g$. When the formula for $Z_{\text {in }}$ is substituted into formula (15) for calculation, the reflection coefficient $\left(S_{11}\right)$ for the designed noncontacting probe circuit can be obtained. If we set formula (15) to be equal to formula (14), the DUT impedance is obtained and set as alphabet $g$. When $g$ is put into the two-port traditional contacting measurement model, as shown in Figure 10, the return loss of DUT is obtained, as formula (15) shows. One has the following:

$$
\begin{gathered}
S_{11}=\frac{Z_{\text {in }}-a}{Z_{\text {in }}+a} \\
h=a \| b \\
i=[(a \| b)+c] \| d \\
j=\{[[(a \| b)+c] \| d]+e\} \| f \\
Q=\frac{(g+50)-50}{(g+50)+50} .
\end{gathered}
$$

As we substitute $S_{11}$ revealed in formula (18) into formula (16), we can obtain the transmission coefficient $\left(S_{21}\right)$. Since the model has a symmetrical circuit, with the voltage division rule as shown in formula (19), it suggests that the $S_{21}$ value can be calculated in the same process from either connector $A$ to point 2 or connector $B$ to point 1 ; also, the DUT impedance, $g$, can be obtained. As shown in Figure 8, when $g$ is put into the two-port traditional contacting measurement model, we can obtain the insertion loss of DUT and that will result in formula (17) as follows:

$$
\begin{gathered}
S_{21}=1+S_{11} \\
Q 1=1+\frac{(g+50)-50}{(g+50)+50} .
\end{gathered}
$$

From the return loss and the insertion loss of the restoring theory, the comparisons of model simulation by agilent advanced design system (ADS) and the 3D EM simulation by the analyzing results of high frequency structure simulator (HFSS), the actual measurement values of DUT can completely be obtained. The two ports of noncontacting probe were used to measure the DUT and transmission line and the results are shown in Figure 9 that the simulation results were close to the measurement ones. In order to confirm that the restoring theory is workable, we changed the DUT from transmission line to the filter (in Figure 10).

3.2. Results. Figures 11 and 12 show the comparisons of the results obtained by the restoring theory, simulation, and measurement. The results of the model simulation are in accordance with those of 3D EM simulation. Nevertheless, the return loss and the insertion loss are mismatched over $7 \mathrm{GHz}$, in which it has larger radiation loss in high frequency. 


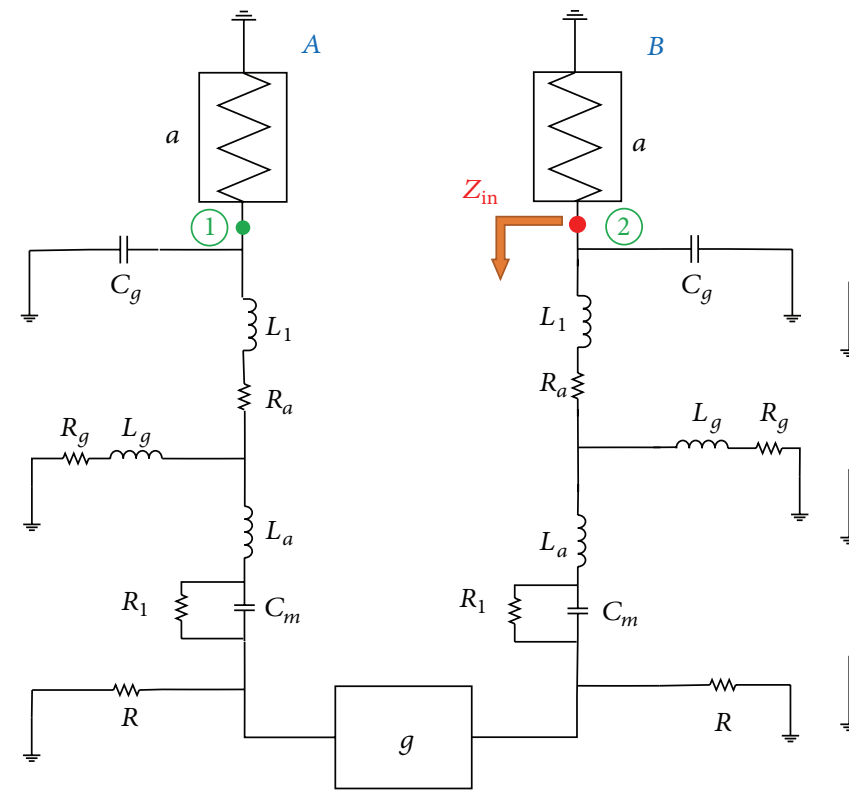

(a)

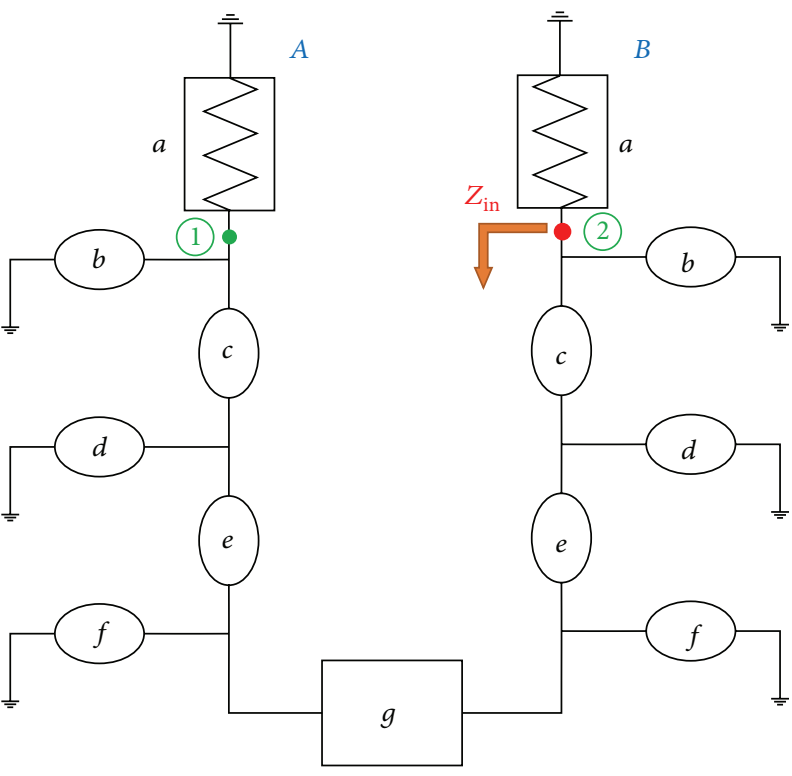

(b)

FIGURE 9: Noncontacting measurement model. (a) Equivalent model with lumped component. (b) Equivalent model with alphabet replaced.

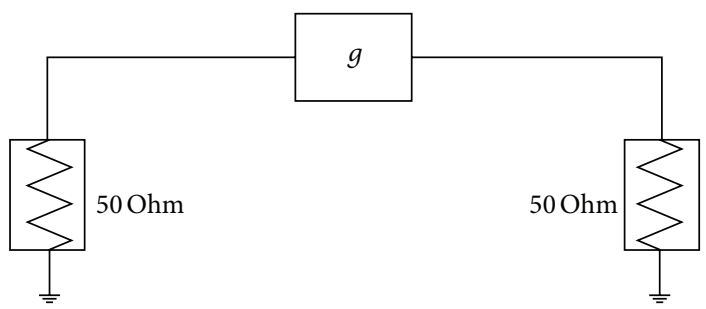

FIGURE 10: Two-port traditional contacting measurement model.

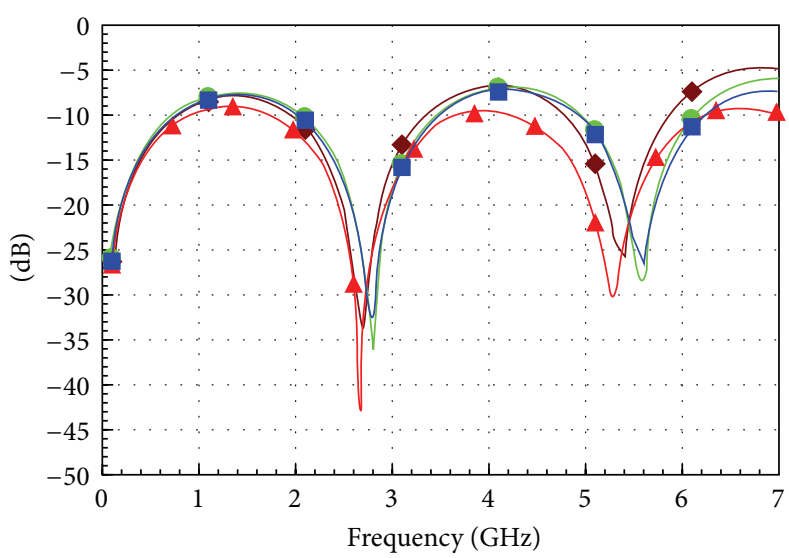

DUT: transmission line $\left(S_{11}\right)$

Simulation_HFSS Modeling

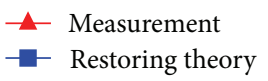

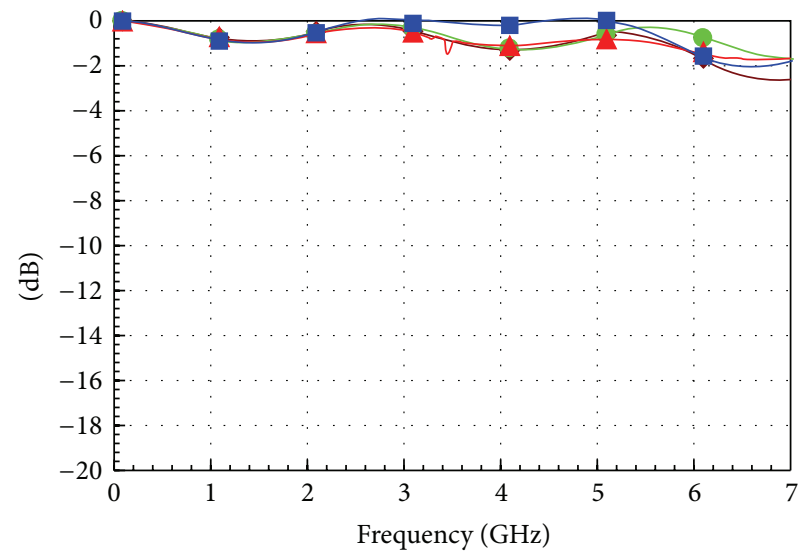

DUT: transmission line $\left(S_{21}\right)$

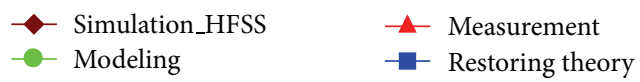

(b)

(a)

FIGURE 11: Results of transmission line reconstruction. (a) Return loss. (b) Insertion loss. 


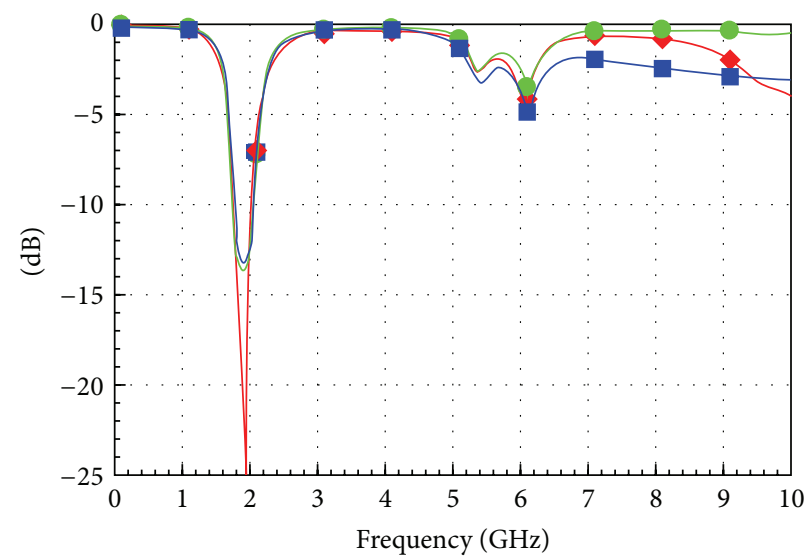

DUT: filter $\left(S_{11}\right)$

- - Simulation_HFSS

- Modeling

Restoring theory

(a)

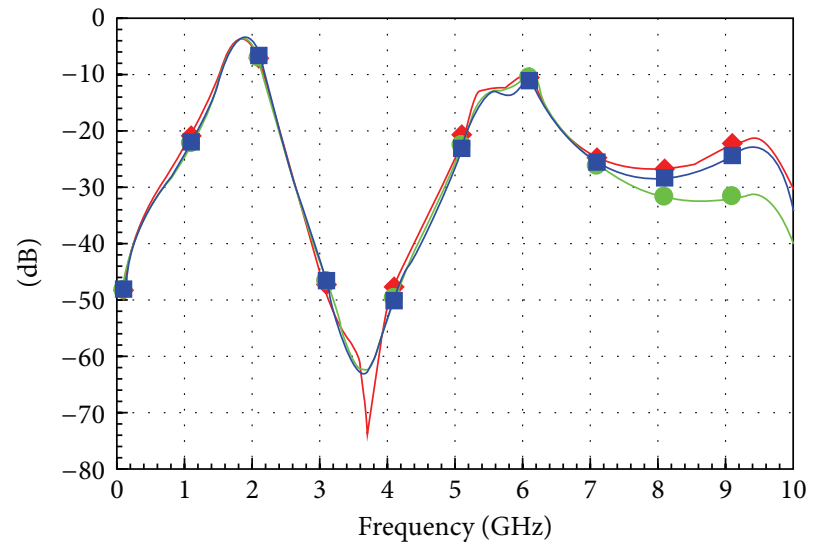

DUT: filter $\left(S_{21}\right)$

- Simulation_HFSS

- Modeling

Restoring theory

(b)

FIGURE 12: Results of filter reconstruction. (a) Return loss. (b) Insertion loss.

Therefore, the feature of a DUT shown in Figures 9 and 10 cannot be completely matched by the results obtained by the lumped elements. Consider

$$
\begin{gathered}
S_{11}=(\{[j(f d+e d+f c+e c+d c)+f e d+f e c+f d c] \\
+g(f d+e d+f c+e c+d c)\}(b-a) \\
-\{[j(f+e+d)+f e+f d] b a \\
+g(f+e+d) b c\}) \\
\times(\{[j(f d+e d+f c+e c+d c)+f e d+f e c+f d c] \\
+g(f d+e d+f c+e c+d c)\}(b+a) \\
-\{[j(f+e+d)+f e+f d] b a \\
+g(f+e+d) b c\})^{-1} \\
+\left(g^{3} 2 b f i j(f d+e d)(f d+e d+f c+e c+d c)\right. \\
+g^{2} 2 b i j[f j(f d+e d+f c+e c+d c)(f d+e d) \\
+f[(j f+j e+f e)(d+c) \\
\quad+(j+f) d c](f d+e d) \\
+d(f d+e d+f c+e c+d c) \\
\times(j f+j e+f e)] \\
\times(f d+e d)+d j(f d+e d+f c+e c+d c) \\
\times(j f+j e+f e)+d[(j f+j e+f e) \\
\\
\quad \times(j f+j e+f e)] \\
\end{gathered}
$$

$$
\begin{aligned}
& +2 b d f j^{2} i[(j f+j e+f e)(d+c)+(j+f) d c] \\
& \times(j f+j e+f e)) \\
& \times\left(g^{4}(f d+e d+f c+e c+d c)\right. \\
& \times[(f d+e d+f c+e c+d c)(a+b) \\
& +a b(f+e+d)](e+f)(i+e) \\
& +g^{3}\{[(f d+e d+f c+e c+d c)(a+b) \\
& +(f+e+d) a b] \\
& \times[(f d+e d+f c+e c+d c)(2 j f+2 j e+f e) \\
& +[(j f+j e+f e)(d+c)+j d c+f d c] \\
& \times(f+e)] \\
& +(f d+e d+f c+e c+d c)(f+e) \\
& \times[[(j f+j e+f e)(d+c)+j d c+f d c](a+b) \\
& +(j f+j e+f e+j d+f d) a b]\}(i+e) \\
& +g^{2}\{[[(j f+j e+f e)(d+c)+j d c+f d c](a+b) \\
& +(j f+j e+f e+j d+f d) a b] \\
& \times[(f d+e d+f c+e c+d c)(2 j f+2 j e+f e) \\
& +[(j f+j e+f e)(d+c)+j d c+f d c] \\
& \times(f+e)] \\
& +[(f d+e d+f c+e c+d c)(a+b) \\
& +(f+e+d) a b]
\end{aligned}
$$




$$
\begin{gathered}
\times[[(j f+j e+f e)(d+c)+j d c+f d c] \\
\times(2 j f+2 j e+f e) \\
+(f d+e d+f c+e c+d c) \\
\times(j f+j e+f e) j]\}(i+e) \\
+g\{[[(j f+j e+f e+j d+f d)(d+c)+j d c+f d c] \\
\times(a+b)+(j f+j e+f e+j d+f d) a b] \\
\times(f d+e d+f c+e c+d c)(j f+j e+f e) j \\
+[(j f+j e+f e+j d+f d)(d+c)+j d c+f d c] \\
\times(2 j f+2 j e+f e)+[(f d+e d+f c+e c+d c) \\
\quad \times(a+b) \\
\quad+(f+e+d) a b] \\
\times[(j f+j e+f e+j d+f d)(d+c)+j d c+f d c] \\
\times(j f+j e+f e) j\}(i+e) \\
+[(j f+j e+f e)(c+d)+(j+f) d c] \\
\times\{[(j f+j e+f e)(c+d)+(j+f) d c] \\
\times(a+b)+[(j f+j e+f e)+j d+f d] a b\} \\
\left.\times(j f+j e+f e) j)^{-1}\right) \\
h+S_{21} \cdot
\end{gathered}
$$

\section{Conclusions}

In this study, a novel noncontact measurement method with the development of using loop-type near-filed probe had been investigated; the transmission lines' frequency responses were measured by this loop-type probe. Another, a radiation model to reconstruct transmission signals had been delivered by equivalent capacitances, inductances, and resistances extracting, too. All parameter formulas in radiation model had been provided and presented. The comparisons of the performance responses among measurement, radiation model, and electromagnetic simulation tool were fitting. We had shown that the measurement method and radiation model presented in this study were useful to evaluate circuit signal performance and EMI/EMS design, like POP, SiP, and subsystem PCB.

\section{Conflict of Interests}

The authors declare that there is no conflict of interests regarding the publication of this paper.

\section{References}

[1] K. Smith, P. Hanaway, M. Jolley et al., "Evaluation of TSV and micro-bump probing for wide I/O testing," in Proceedings of the
IEEE International Test Conference (ITC '11), pp. 1-10, September 2011.

[2] K. Soejima, M. Kimura, Y. Shimada, and S. Aoyama, "New probe microstructure for full-wafer, contact-probe cards," in Proceedings of the 49th Electronic Components and Technology Conference, pp. 1175-1180, June 1999.

[3] J. T. Huang, K. Y. Lee, C. S. Wu, C. Y. Lin, and S. H. Shih, "Using micro-electroforming and micro-assembly technology to fabricate vertical probe card," in Proceedings of the International Conference on Electronic Materials and Packaging (EMAP '06), pp. 1-5, December 2006.

[4] Y.-C. Tsao, J.-J. Tang, and Y.-L. Hsieh, "Analysis of probing effects on solder bump," in Proceedings of the 6th Electronics Packaging Technology Conference (EPTC '04), pp. 303-307, December 2005.

[5] S. Kumar, S. Rao, T. K. Guan, and F. Harun, "Influence of wafer probing against initial bonding," in Proceedings of the 34th International Electronics Manufacturing Technology Conference (IEMT '10), December 2010.

[6] J. Y. Kim, H. J. Lee, H. J. Choi, S. J. Lee, and S. W. Moon, “Design, fabrication and mechancial characterization of vertical micro contact probe," in Proceedings of the 5th IEEE Conference on Sensors, pp. 1155-1158, Daegu, Republic of Korea, October 2006.

[7] M. Zapatka and R. Ziser, "An introduction to coaxial RF probing solutions for mass-production tests," in Proceedings of the 74th ARFTG Microwave Measurement Symposium, pp. 1-6, December 2009.

[8] J. Andes and E. Bogatin, "The socket response to current packaging and test trends," in Proceedings of the IEEE/CPMT/SEMI 29th International Electronics Manufacturing Technology Symposium, pp. 97-99, July 2004.

[9] S.-K. Hung, C.-F. Tsai, Y.-P. Hsu, D.-J. Tzou, M.-H. Lin, and L.-C. Fu, "Automatic probe alignment for atomic force microscope," in Proceedings of the IEEE International Conference on Mechatronics (ICM '05), pp. 909-912, July 2005.

[10] S.-Y. Lin, S.-K. Yen, W.-S. Chen, and P.-H. Cheng, "Printed magnetic field probe with enhanced performances," in Proceedings of the Asia Pacific Microwave Conference (APMC '09), pp. 649652, December 2009.

[11] J.-M. Kim, W.-T. Kim, and J.-G. Yook, "Resonance-suppressed magnetic field probe for em field-mapping system," IEEE Transactions on Microwave Theory and Techniques, vol. 53, no. 9, pp. 2693-2699, 2005.

[12] H. Funato and T. Suga, "Magnetic near-field probe for $\mathrm{GHz}$ band and spatial resolution improvement technique," in Proceedings of the 17th International Zurich Symposium on Electromagnetic Compatibility (EMC-Zurich '06), pp. 284-287, March 2006.

[13] C. H. Huang, R. F. Hsu, S. M. Wu, Y. C. Tang, and C. C. Chen, "Radiation modeling and performance reconstructing of signal connection in package substrate using non-contacting probe," in Proceedings of the Asia-Pacific Microwave Conference (APMC '11), pp. 1035-1038, December 2011.

[14] J.-K. Wang, R.-F. Hsu, W.-T. Chien, S.-M. Wu, C.-C. Chen, and M.-S. Lin, "Vertical signal transmission by non-contact technology application for 3DIC," in Proceedings of the AsiaPacific Microwave Conference (APMC '13), pp. 827-829, November 2013.

[15] H. A. Kalhor, "Comparison of Ampere's circuital law (ACL) and the law of Biot-Savart (LBS)," IEEE Transactions on Education, vol. 31, no. 3, pp. 236-238, 1988.

[16] D. K. Cheng, Field and Wave Electromagnetics, Addison-Wesley, New York, NY, USA, 2nd edition, 1989. 


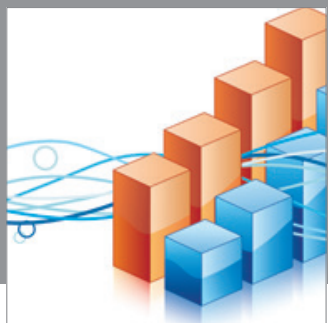

Advances in

Operations Research

mansans

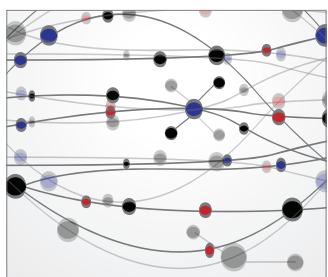

The Scientific World Journal
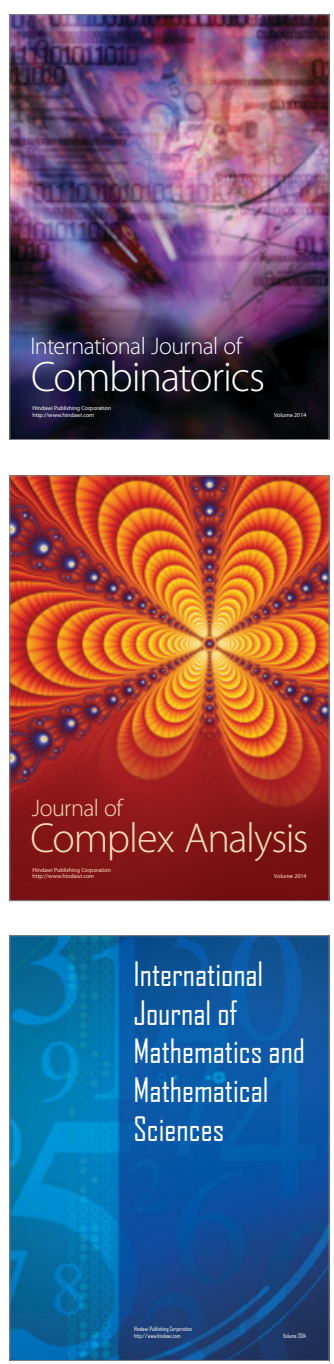
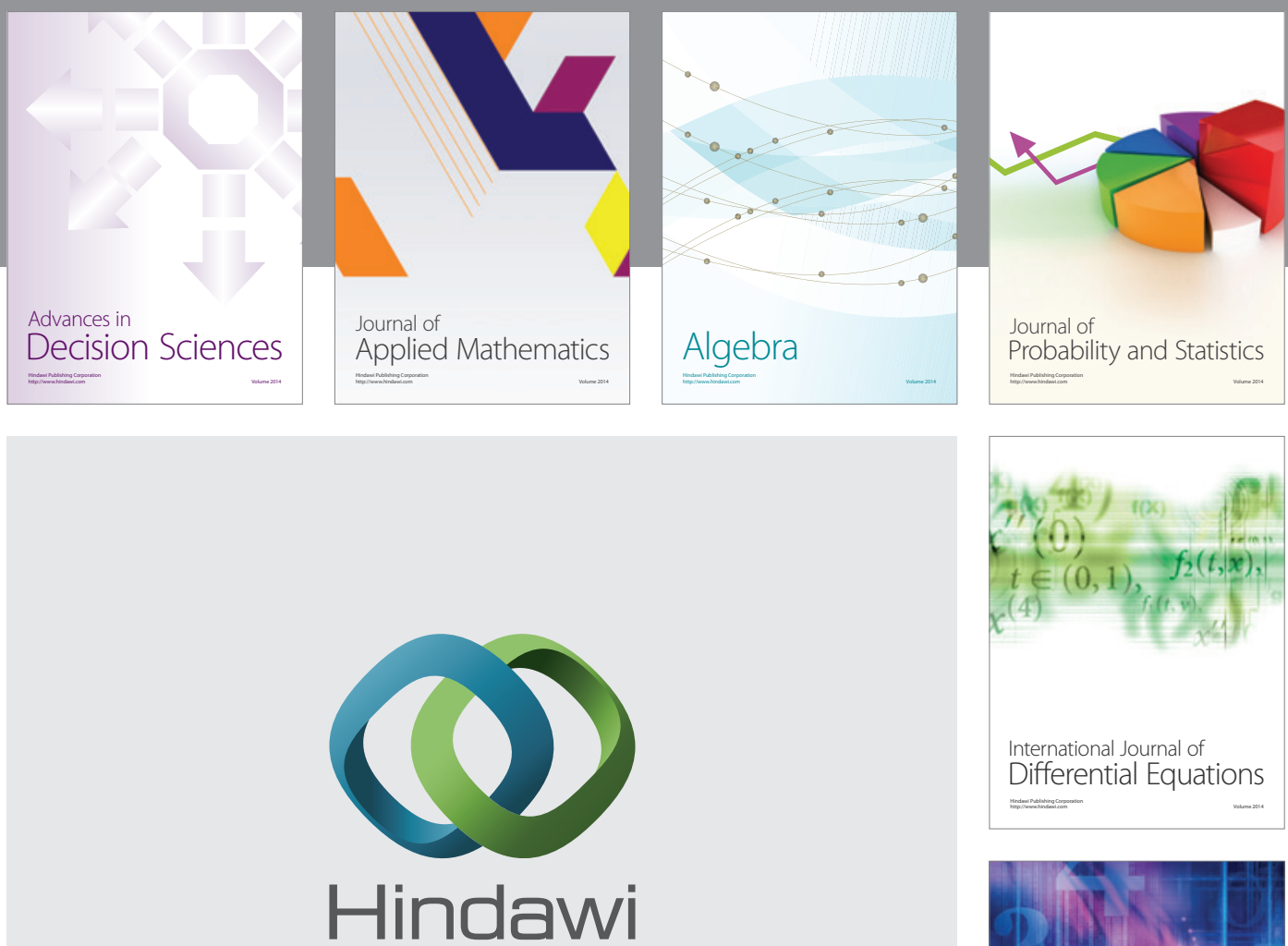

Submit your manuscripts at http://www.hindawi.com
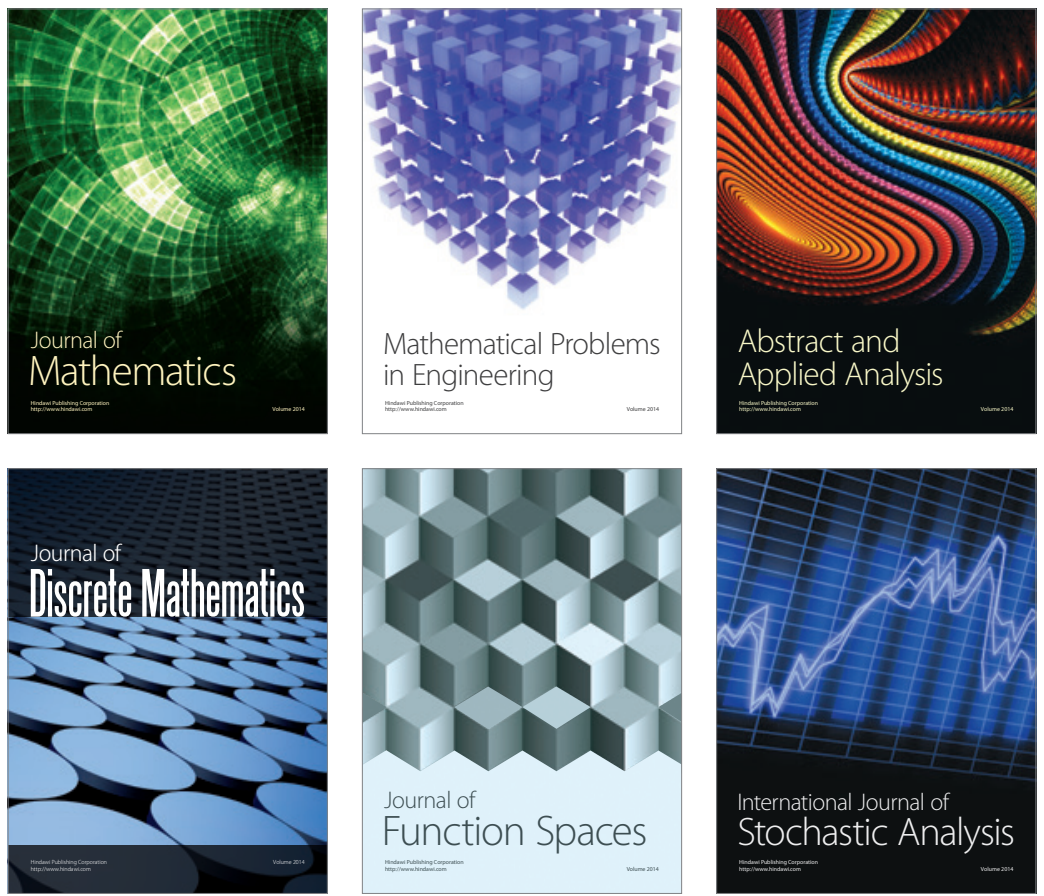

Journal of

Function Spaces

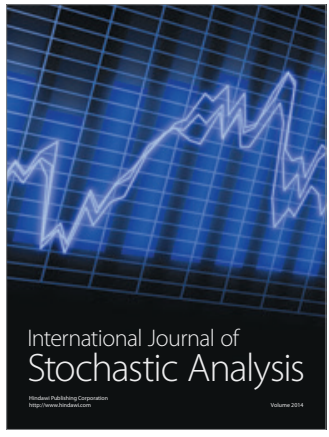

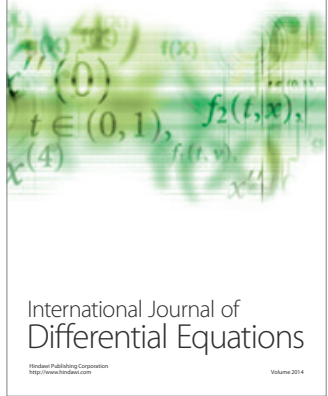
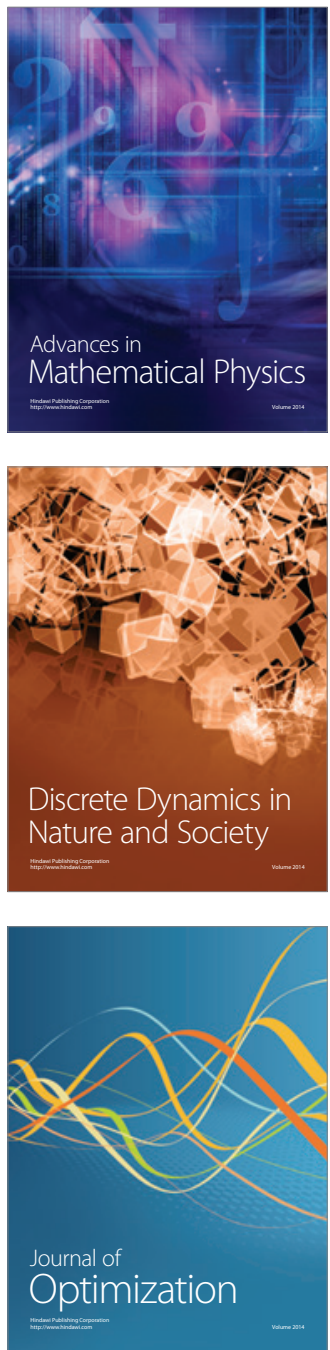